\title{
Opposing Gradients of Ribbon Size and AMPA Receptor Expression Underlie Sensitivity Differences among Cochlear-Nerve/Hair-Cell Synapses
}

\author{
Leslie D. Liberman, ${ }^{1}$ Haobing Wang, ${ }^{1}$ and M. Charles Liberman ${ }^{1,2,3}$ \\ ${ }^{1}$ Eaton-Peabody Laboratory, Massachusetts Eye \& Ear Infirmary, Boston, Massachusetts 02114-3096, ${ }^{2}$ Department of Otology and Laryngology, Harvard \\ Medical School, Boston, Massachusett 02114, and 3Program in Speech and Hearing Biosciences and Technology, Harvard-Massachusetts Institute of \\ Technology, Cambridge, Massachusetts 02114
}

The auditory system transduces sound-evoked vibrations over a range of input sound pressure levels spanning six orders of magnitude. An important component of the system mediating this impressive dynamic range is established in the cochlear sensory epithelium, where functional subtypes of cochlear nerve fibers differ in threshold sensitivity, and spontaneous discharge rate (SR), by more than a factor of 1000 (Liberman, 1978), even though, regardless of type, each fiber contacts only a single hair cell via a single ribbon synapse. To study the mechanisms underlying this remarkable heterogeneity in threshold sensitivity among the 5-30 primary sensory fibers innervating a single inner hair cell, we quantified the sizes of presynaptic ribbons and postsynaptic AMPA receptor patches in $>1200$ synapses, using high-power confocal imaging of mouse cochleas immunostained for CtBP2 (C-terminal binding protein 2, a major ribbon protein) and GluR2/3 (glutamate receptors 2 and 3). We document complementary gradients, most striking in mid-cochlear regions, whereby synapses from the modiolar face and/or basal pole of the inner hair cell have larger ribbons and smaller receptor patches than synapses located in opposite regions of the cell. The AMPA receptor expression gradient likely contributes to the differences in cochlear nerve threshold and SR seen on the two sides of the hair cell in vivo (Liberman, 1982a); the differences in ribbon size may contribute to the heterogeneity of EPSC waveforms seen in vitro (Grant et al., 2010).

\section{Introduction}

All information about the acoustic environment is carried from the inner ear to the brainstem by the afferent fibers of the cochlear nerve, most of which (95\%) receive synaptic input only from inner hair cells (Spoendlin, 1969; Liberman, 1982a). Each of these myelinated, bipolar sensory neurons sends a peripheral projection to a single inner hair cell (IHC) via a single unmyelinated terminal, forming a single ribbon synapse (Liberman, 1980a; Liberman et al., 1990) (Fig. 1), at which transmitter release acts postsynaptically on AMPA-type glutamate receptors (GluRs) in the cochlear nerve terminals (Matsubara et al., 1996; Ruel et al., 2007; Meyer et al., 2009; Grant et al., 2010). The position of the IHC along the mechanically tuned cochlear spiral determines frequency selectivity; fibers from the apical turn are tuned to low frequencies, those from the basal turn to higher frequencies (Liberman, 1982b). Intracellular labeling studies suggested that the position of the synapse around the hair cell circumference determines the fibers' threshold sensitivity: fibers contacting the "pillar face" of the IHC [closer to the outer hair cells (OHCs)]

\footnotetext{
Received June 30, 2010; revised 0ct. 19, 2010; accepted 0ct. 22, 2010.

This work was supported by Grants R01 DC0188 and P30 DC5029 from the National Institute on Deafness and other Communication Disorders.

Correspondence should be addressed to Dr. M. Charles Liberman, Eaton-Peabody Laboratory, Massachusetts Eye and Ear Infirmary, 243 Charles Street, Boston, MA 02114-3096. E-mail: charles_liberman@meei.harvard.edu. DOI:10.1523/JNEUROSCI.3389-10.2011

Copyright $\odot 2011$ the authors $\quad 0270-6474 / 11 / 310801-08 \$ 15.00 / 0$
}

have lower thresholds to acoustic stimulation [and higher spontaneous discharge rates (SRs)]; those on the opposite, "modiolar," face of the IHC have higher acoustic thresholds and low SRs (Liberman, 1982a). The differences in central projections of low-SR versus high-SR fibers underscore the functional significance of this physiological classification (Liberman, 1991). In addition to expanding the cochlea's dynamic range, the highthreshold, low-SR group's resistance to masking noise is likely critical for hearing in a noisy environment (Costalupes et al., 1984).

The mechanisms underlying a threshold difference of up to three orders of magnitude $(60 \mathrm{~dB})$ between nerve fibers synapsing on different sides of the sensory receptor has remained a mystery. Ultrastructural studies reveal no intracellular compartmentalization presynaptically to suggest that receptor potentials could differ on the two sides of the sensory cell (Liberman, 1980a). Serial section reconstruction of IHCs in the cat showed that pillar-side fibers are larger in caliber and richer in mitochondria than those on the modiolar face; however, this postsynaptic difference is more likely an effect of, rather than the cause of, differences in SR and threshold sensitivity (Liberman, 1980a): certainly, fibers discharging spontaneously at $>100 \mathrm{sp} / \mathrm{s}$ have higher metabolic requirements than those that are quiescent in the absence of sound.

In the present study, we quantitatively re-examine the spatial organization of afferent synapses on sensory cells in the mouse cochlea. By immunostaining and high-power confocal micros- 
copy of epithelial whole mounts, coupled with novel threedimensional (3-D) image-analysis tools, we document opposing gradients of ribbon size and AMPA receptor (AMPAR) expression from a large number of synapses sampled from IHCs all along the cochlear spiral. We show that afferent synapses closer to the IHC's modiolar side and/or basal pole have larger ribbons and smaller receptor patches, whereas synapses on the pillar face and/or closer to the hair bundle have smaller ribbons and larger receptor patches. The differences in presynaptic ribbon size may contribute to the heterogeneity of EPSC shapes observed in vitro (Grant et al., 2010); the spatial gradient in postsynaptic AMPA receptor patches likely underlies the fundamental sensitivity differences observed in vivo among the cochlear nerve fibers innervating a single sensory cell (Liberman, 1978).

\section{Materials and Methods}

\section{Immunostaining and dissection protocols}

CBA/CaJ mice, aged 6-9 weeks, were used for all experiments. AMPA receptor labeling was successful only with minimally fixed tissue. After anesthetization with ketamine, animals were decapitated and cochleas quickly removed to cold PBS. Round and oval windows were opened, and bone over the apical turn removed to allow rapid flushing of $4 \%$ paraformaldehyde in PBS through the cochlear scalae followed by brief $(10 \mathrm{~min})$ postfixation at $4^{\circ} \mathrm{C}$. After postfixation, cochleas were transferred back into PBS, and fine forceps were used to remove more of the thin bone over the middle-ear-facing portion of the cochlear spiral and to pull off the tectorial membrane. Without decalcification or further dissection, cochleas were immediately immunostained in toto, by incubating in PBS with $1 \%$ Triton X-100, plus (1) $5 \%$ normal horse serum for $1 \mathrm{~h}$; then (2) primary antibodies [rabbit anti-GluR2/3 from Millipore Bioscience Research Reagents at 1:100 and mouse anti-C-terminal binding protein (CtBP2) from BD Transduction Laboratories at 1:200] for $\sim 20 \mathrm{~h}$ at $37^{\circ} \mathrm{C}$; followed by (3) chicken anti-rabbit coupled to Alexa Fluor488 at 1:1000 and biotinylated donkey anti-mouse at 1:200 for $1 \mathrm{~h}$ at $37^{\circ} \mathrm{C}$; and finally (4) goat anti-chicken coupled to Alexa Fluor 488 at 1:1000 and streptavidin coupled to Alexa Fluor 568 at 1:1000 for $1 \mathrm{~h}$ at $37^{\circ} \mathrm{C}$. At the start of the primary incubation, some ears were subjected to seven cycles of microwave radiation at $150 \mathrm{~W}$ (on for $3 \mathrm{~min}$, off for 3 $\min$ ). Following the final antibody linkage step, the tissue was further fixed by immersion in $4 \%$ paraformaldehyde in PBS for $30 \mathrm{~min}$ on a shaker at room temperature. After immunostaining, cochleas were decalcified in $0.12 \mathrm{~m}$ EDTA overnight at $4^{\circ} \mathrm{C}$ and dissected into five or six pieces, mounted on microscope slides in VectaShield, and coverslipped.

For immunohistochemistry when glutamate receptor labeling was not required, animals were anesthetized with ketamine and then intravascularly perfused with $4 \%$ paraformaldehyde in PBS. Following perfusion, bullas were opened to expose the cochlea, and round and oval windows were opened to permit flushing of fix through the scalae. Cochleas were then removed and postfixed in the same fixative for $2.5 \mathrm{~h}$, and finally decalcified in EDTA. Microdissected pieces were then immunostained much as described above. Additional primary antibodies used were as follows: (1) goat anti-NKA $\alpha 3$ (sodium/potassium ATPase $\alpha 3$ subunit from Santa Cruz Biotechnology at 1:100) to stain terminals of cochlear nerve fibers; and (2) rabbit anti-myosin VIIA (from Proteus at 1:200) to stain IHCs.

To examine possible spatial gradients in the expression of presynaptic voltage-gated calcium channels (Frank et al., 2009), we immunostained for CaV1.3 but were unable to get a useful signal with fixation protocols that allow complete cochlear dissection. Successful staining in mouse requires brief methanol fixation, which only reliably yields small pieces of the most apical turn.

\section{3-D morphometry}

Cochlear lengths were measured for each piece from each case, and a cochlear frequency map computed (Muller et al., 2005) to precisely localize hair cells from different frequency regions. Confocal $z$-stacks from selected cochlear regions from each ear were obtained on a Leica TCS SP2 using a high-resolution (1.4 numerical aperture) oil-immersion objec- tive and $4 \times$ digital zoom. Images were acquired in a $1024 \times 1024$ raster (pixel size $=0.036 \mu \mathrm{m}$ in $x$ and $y$ ) with $0.122 \mu \mathrm{m}$ steps in $z$. Care was taken to minimize pixel saturation in each image stack by using the display feature that highlights 0 's and 256's in the image as green and blue, respectively (supplemental Fig. 1, available at www.jneurosci.org as supplemental material).

Each stack contained the entire synaptic pole of four to six inner hair cells as viewed from the endolymphatic surface of the organ of Corti (Fig. 1). Each stack contained the synapses of 40 to 120 cochlear nerve fibers, depending on cochlear location (Kujawa and Liberman, 2009). Image stacks were ported to an off-line processing station, where further 3-D morphometry was performed using a commercial image-processing program (Amira, Visage Imaging) and custom software written in $\mathrm{C}++$ or LabVIEW. All quantitative analysis was performed on the raw image stacks, without deconvolution, filtering, gamma correction, or resampling.

Elemental volumes. In Amira, the volumes of all presynaptic ribbons (red channel) and postsynaptic AMPA receptor patches (green channel) were computed using functions that (1) allow 3-D rendering and visualization of "isosurfaces" enveloping all pixel clusters with intensities greater than a user-defined criterion value (and with greater than a minimum number of enveloped pixels) and then (2) measure the volumes of all clusters so identified along with the $x, y$, and $z$ coordinates of their centers. Isosurface pixel values were typically 80 (of 256) for the red (synaptic ribbon) channel and 40 (of 256) for the green (AMPA receptor patches) channel. To normalize for differences in staining intensity across cases and cochlear regions, all volumes from each $z$-stack were normalized by dividing by the median volume for all the pixel clusters (containing both modiolar /basal and pillar/apical zones) in that channel and in that $z$-stack. Median, rather than mean, values were used to minimize contribution from a small number of very large elements in some distributions, which may represent staining artifacts.

Colocalization. Using the independently derived $x, y$, and $z$ coordinates of all ribbons and receptor patches in each $z$-stack, custom software written in $\mathrm{C}++$ was used to produce, for each ribbon or receptor patch, a thumbnail image of the $x y$ projection of a voxel cube (1.5-2.0 $\mu \mathrm{m}$ on a side), extracted from the confocal image stack, centered on each ribbon or receptor patch. The numbers of colocalized versus solitary ribbons and/or receptor patches were determined by visual inspection of these thumbnail arrays (see Fig. 3).

Spatial segregation around the hair cell. The $x, y$, and $z$ coordinates of all ribbons and receptor patches from each confocal $z$-stack were ported to custom LabVIEW software where $y z$ coordinates of the synapse locations could be remapped into an $x y$ plane and analyzed (simulating a crosssectional view of the organ of Corti, as in Fig. 1), with ribbon and receptor-patch size rendered as the $z$-dimension of the plot. The software interface allowed rotation and/or translation of the new $x y$ coordinate system to reorient the virtual hair cell until two groups of synapses, with different ribbon/receptor-patch sizes, were optimally separated by the $y$-axis into the following two groups: one clustered on the modiolar face and/or basal pole of the cell; and a second clustered on the pillar face and/or apical pole of the cell.

\section{Results}

\section{Afferent innervation of the inner hair cell}

In the mammalian inner ear, 95\% of cochlear nerve fibers synapse only with IHCs. The innervation pattern is punctate, with each neuron contacting a single IHC by a single terminal swelling, and each IHC forming synapses with 5-30 cochlear nerve fibers, depending on species and cochlear location (Liberman et al., 1990). As shown in Figure 1, the ultrastructure of this afferent synapse consists of an electron-dense presynaptic ribbon in the IHC, surrounded by a halo of synaptic vesicles, and a discrete plaque of presynaptic and postsynaptic membrane density (Merchan-Perez and Liberman, 1996).

In the present study, we used an antibody to a component of the ribeye protein CtBP2 to immunostain synaptic ribbons (Khimich et al., 2005) and an antibody to an isoform of the 


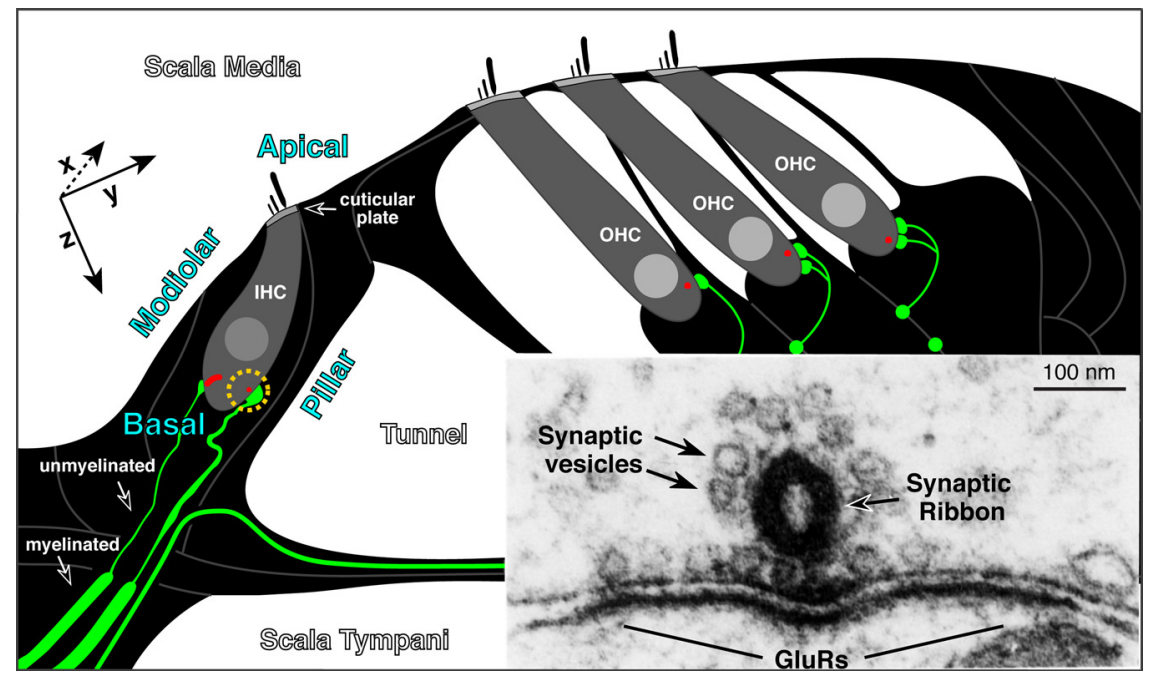

Figure 1. Schematic cross section through the cochlear epithelium showing the unmyelinated afferent terminals (green) on $\mathrm{IHCs}$ and $\mathrm{OHCs}$ and the presynaptic ribbons at each synapse (red). The modiolar and pillar side of the IHC, its apical versus basal pole, and the position of the cuticular plate are indicated. Inset shows an electron micrograph (Liberman, 1980b) of the presynaptic ribbon in an IHC, its halo of synaptic vesicles, and the postsynaptic membrane density on the terminal swelling, where GluRs are located. Approximate orientation of $x-, y$-, and $z$-planes in the subsequent confocal images is shown: images are acquired from epithelial whole mounts, viewed from the scala media surface, thus the $x$-axis runs into, and out of, the plane of the schematic.

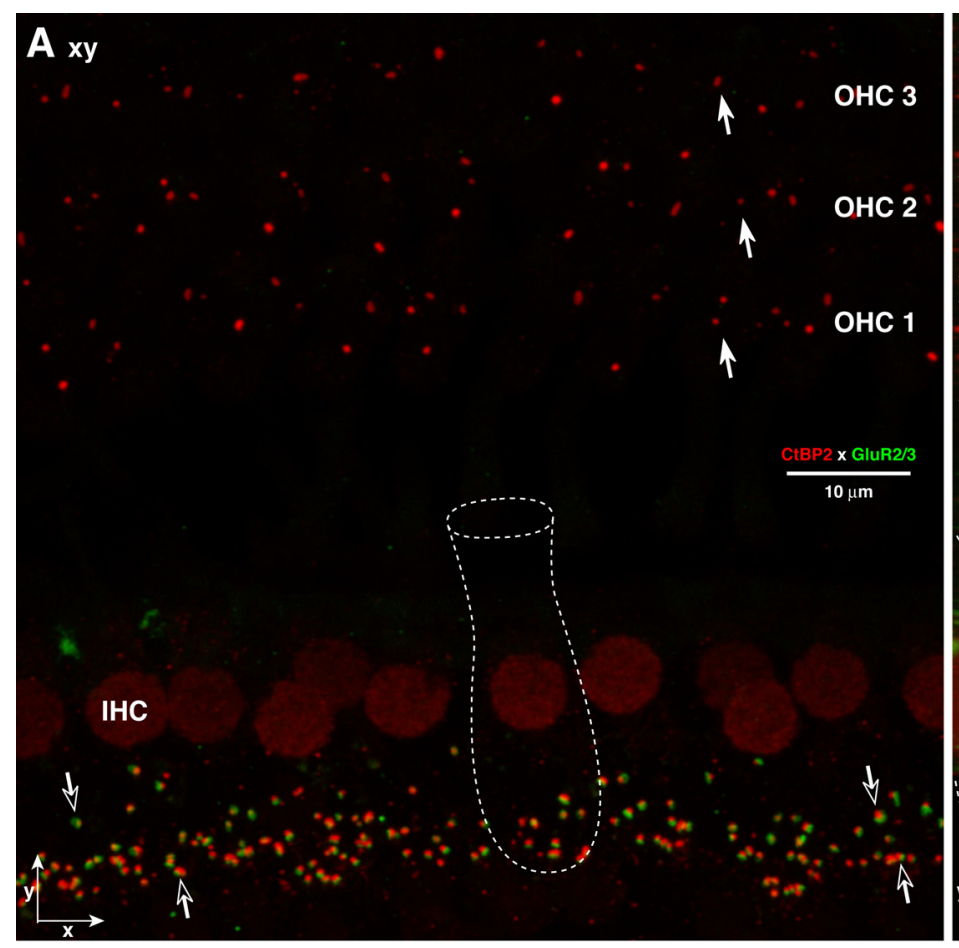

Figure 2. Afferent synapses on $\mathrm{HCC}$ and $\mathrm{OHCs}$ are seen by immunostaining presynaptic ribbons with anti-CtBP2 (red) and postsynaptic densities with anti-GluR2/3 (green). In the IHC area, most synapses include closely apposed red and green puncta (open arrows), and IHC nuclei are also weakly immunopositive for $\mathrm{CtBP2}$. In the $\mathrm{OHC}$ area, only $\mathrm{CtBP2}$-positive puncta are seen (filled arrows). $\boldsymbol{A}$, An xy projection of a confocal $z$-stack through the synaptic regions of $12 \mathrm{lHCs}$ and numerous $0 \mathrm{HCs}$ from the $8 \mathrm{kHz}$ region. $\boldsymbol{B}$, a yz projection of the same $z$-stack. Outlines (approximate) of individual hair cells are shown by dotted white lines in $\boldsymbol{A}$ and $\boldsymbol{B}$.

$\mathrm{Na}^{+} / \mathrm{K}^{+}$ATPase (NKA $\alpha 3$ ) (McLean et al., 2009) to immunostain unmyelinated cochlear nerve terminals within the sensory epithelium. Counts of CtBP2-positive puncta in normal mouse cochleas yield an average of 16.8 ribbons per IHC in middle of the cochlear spiral, which corresponds precisely to synaptic counts

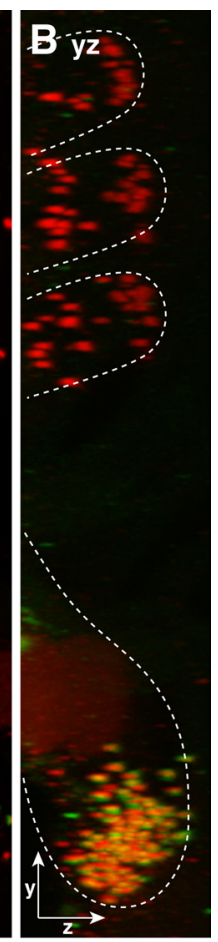

derived from a serial section ultrastructural study in mouse (Stamataki et al., 2006). Double staining for ribbons and terminals shows a nearly perfect one-forone relation in the IHC area (supplemental Fig. 2, available at www.jneurosci.org as supplemental material): every ribbon is intimately associated with a terminal swelling, and every labeled swelling is apposed to a presynaptic ribbon, as expected from the ultrastructural serial section data (Liberman, 1980a; Stamataki et al., 2006).

\section{Spatial segregation of synaptic} morphologies: qualitative analysis

To label postsynaptic densities on cochlear nerve terminals, we immunostained with an antibody to AMPA-type glutamate receptors that recognizes both GluR2 and GluR3 subunits (recently renamed GluA2 and GluA3) (Collingridge et al., 2009). The AMPAR immunolabeling was only successful with minimal fixation, and when all antibody incubations were performed before decalcification (see Materials and Methods). In confocal image stacks (Fig. 2), small AMPAR-positive patches are plentiful in the subnuclear region of the IHCs, where they appear in close proximity to CtBP2-positive synaptic ribbons. In contrast, in the OHC area only synaptic ribbons are seen, and no AMPAR-positive patches are visible.

High-power analysis of double-stained epithelia revealed a nearly perfect onefor-one relation between AMPAR patches and synaptic ribbons in the IHC area: as shown in the thumbnail projection array of Figure 3, containing an $x y$ image centered on each of the ribbons from five adjacent IHCs, almost every ribbon is intimately associated with a patch of AMPAR labeling, with relative position and size consistent the ultrastructural pictures of these presynaptic and postsynaptic elements shown in Figure 1. Similar near-perfect correspondence was seen from independently generated image arrays with thumbnails centered on every AMPAR-positive patch (data not shown). The thumbnail array in Figure 3 is ordered (top to bottom and left to right) by decreasing ribbon volume, thereby revealing clear opposing gradients: the largest ribbons (left columns) tend to have the smallest AMPAR patches, and the smallest ribbons (right columns) have the largest AMPAR patches.

Three-dimensional analysis of synaptic morphology versus synaptic position on the IHC membrane revealed a striking spatial organization as well. As seen from the epithelial surface (i.e., the $x y$ projection) (Fig. $4 \mathrm{~A}$ ), the synapses farthest from the OHCs tend to have large ribbons and small AMPAR patches, and those 
closest to the OHCs tend to have smaller ribbons and larger AMPAR patches. After rotating the image stack to view the projection as a cross section through the epithelium (i.e., the $y z$ plane) (Fig. $4 B$ ), the segregation is even clearer. As shown by the dashed yellow arc, synapses at the basal pole of the IHC tend to have large ribbons and small AMPAR patches; those closer to the hair bundle (apical pole) tend to have small ribbons and large AMPAR patches. Given that prior ultrastructural studies suggesting functional segregation of neuronal types around the IHC circumference (Liberman, 1980a) used sections cut parallel to the cuticular plate (Fig. 1), and that the IHC body has a pronounced tilt in regard to the cuticular plate, the solid yellow line in Figure $4 B$ approximately divides the modiolar and pillar faces of the IHC, as defined in prior studies. Recognizing the nomenclature ambiguities that result from the $\sim 45^{\circ}$ inclination of the IHC within the sensory epithelium, in the results that follow we lump the modiolar face with the basal pole and the pillar face with the apical pole of the synaptic region of the IHC.

To ensure that the spatial trends were not an artifactual difference in fluorescent signal strength arising from differences in tissue depths within the relatively thick cochlear whole mounts, we analyzed ribbon sizes in sectioned material and showed that the same trends are visible even when material is viewed directly in cross section (supplemental Fig. 3, available at www. jneurosci.org as supplemental material). The existence of opposing gradients of ribbon size and AMPAR patch size also argues strongly against either gradient reflecting an artifact of differential signal penetration. We also observed the same gradient of ribbon size in IHCs from adult guinea pig (supplemental Fig. 5, available at www.jneurosci.org as supplemental material); GluR2/3 immunostaining was not attempted.

\section{Spatial segregation of synaptic} morphologies: quantitative analysis To quantify the spatial organization of synaptic morphologies, we used imageprocessing software to measure the volume of the ribbons and AMPAR patches from 1243 synapses (i.e., colocalized ribbons and AMPAR patches) from 22 confocal $z$-stacks comprising $\sim 100$ IHCs and spanning the 2.0 to the $50 \mathrm{kHz}$ regions of the cochlea, in tissue obtained from three different ears. To normalize for differences in staining intensity between tissue samples, the element volumes in each $z$-stack were normalized to the median size for that element type (i.e., ribbon or AMPAR patch) in that $z$-stack. To segregate synapses into two groups, the $y z$ projection from each $z$-stack (Fig. $4 B$ ) was rotated until a vertical line could be placed to optimize the segregation of synaptic morphol-

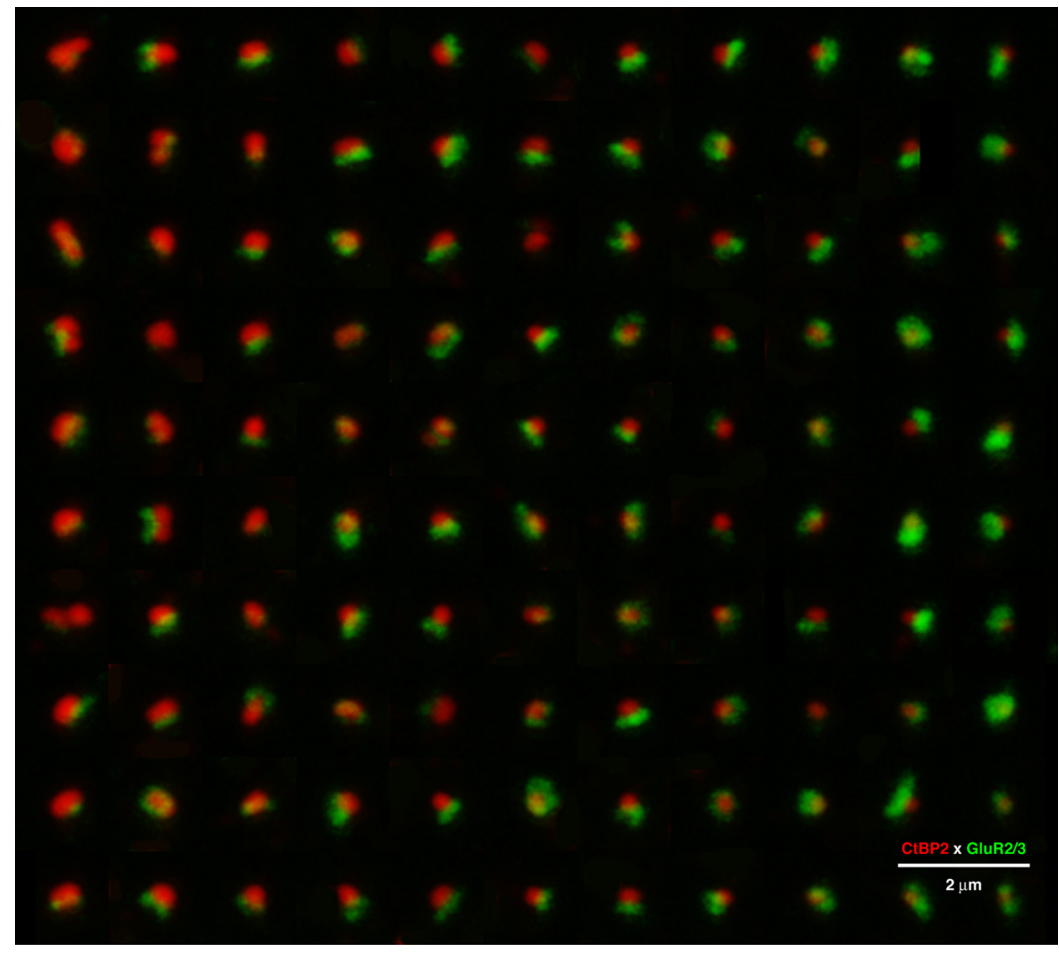

Figure 3. The opposing size gradients of presynaptic and postsynaptic elements are seen by arraying images of all the afferent synapses from five adjacent inner hair cells in exact descending order of ribbon volume, defined using a criterion brightness of 50 (of 256) in the red channel. Larger ribbons (top left) are associated with small AMPAR patches, and small ribbons (bottom right) are centered on a different ribbon. Each ribbon from the $z$-stack is shown exactly once: for some thumbnails, off-center (nearby) ribbons have been erased for clarity. For display, the image array was resampled (bicubic) in Photoshop to minimize pixelization: quantification was always performed on raw image stacks. These data were obtained from the $16 \mathrm{kHz}$ region.
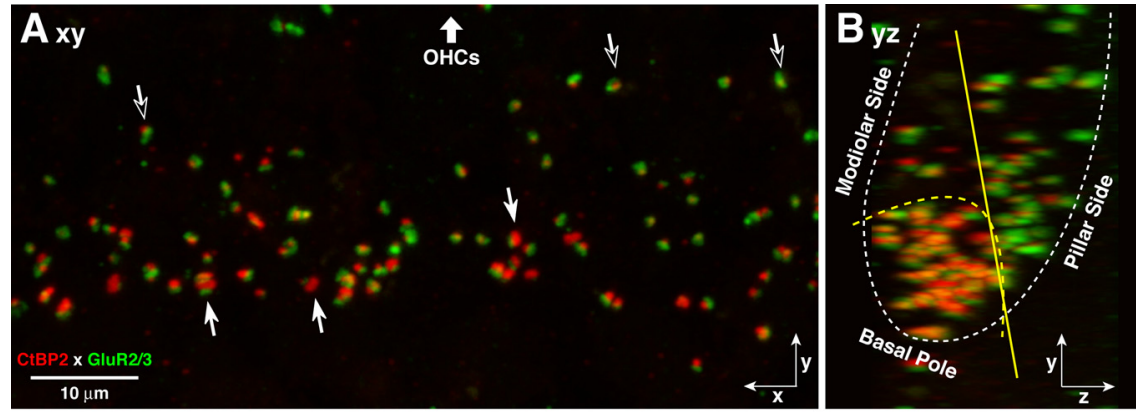

Figure 4. $\quad$ A, Afferent synapses on inner hair cells show opposing spatial gradients in size of the presynaptic and postsynaptic elements: in the xy projection, synapses with the largest ribbons and smallest AMPAR patches are farthest from the OHCs (filled arrows), while those closer to the $\mathrm{OHCs}$ (open arrows) tend to have large AMPAR patches and small ribbons. $\boldsymbol{B}$, In the $y z$ projection, the largest ribbons are on the modiolar side and basal pole of the hair cell, while the largest GluR2/3 patches are on the pillar side: the approximate outline of the IHCs is shown by dotted white lines. This confocal z-stack is through the synaptic regions of four adjacent IHCs from the $14 \mathrm{kHz}$ region. See text for explanation of the yellow lines in $\boldsymbol{B}$.

ogies (Fig. 4B). As shown Figure 5, the resultant separation yielded fewer synapses in the pillar/apical zone (510 or $\sim 40 \%)$ than in the modiolar/basal zone ( 733 or $\sim 60 \%$ ), in keeping with results from prior ultrastructural studies (Liberman, 1980a).

The ensemble histograms (Fig. 5A,B) show that ribbon volumes across all cochlear regions were greater in the modiolar/ basal zone than in the pillar/apical zone, whereas AMPAR patches showed the opposite trend. Both sets of differences were highly significant by unpaired Student's $t$ test ( $p \ll 0.01$ ). The mean (or median) data showed that AMPAR patches were $~ 64 \%$ (or 70\%) bigger in the pillar/apical zone while synaptic ribbons 

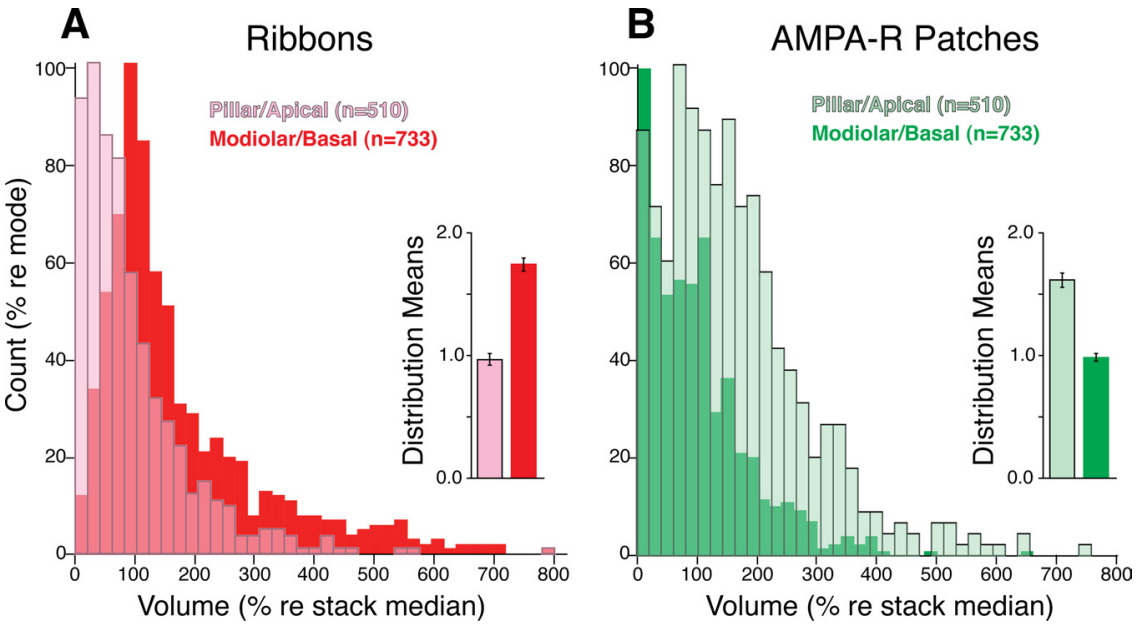

Figure 5. $A, B$, Spatial segregation of synaptic morphologies on the sensory cell: synapses in the pillar/apical zone of the IHC have larger AMPAR patches $(\boldsymbol{B})$ and smaller presynaptic ribbons $(\boldsymbol{A})$ than those in the modiolar/basal region. Histograms represent data from 1243 colocalized CtBP2- and GluR2/3-positive puncta pooled from 22 confocal $z$-stacks from three different ears (Fig. 4 shows puncta from one $z$-stack). Each image stack included the synaptic poles of four to five adjacent inner hair cells. Data were obtained from all cochlear turns, from the 4 to the $55 \mathrm{kHz}$ region (see Fig. 6). Inset in each panel shows the mean volume of ribbons $(\boldsymbol{A})$ or AMPAR patches $(\boldsymbol{B})$ for the two regions of the IHC. Error bars in the insets are SEMs computed across all ribbons or AMPAR patches. Further technical details can be found in Materials and Methods.

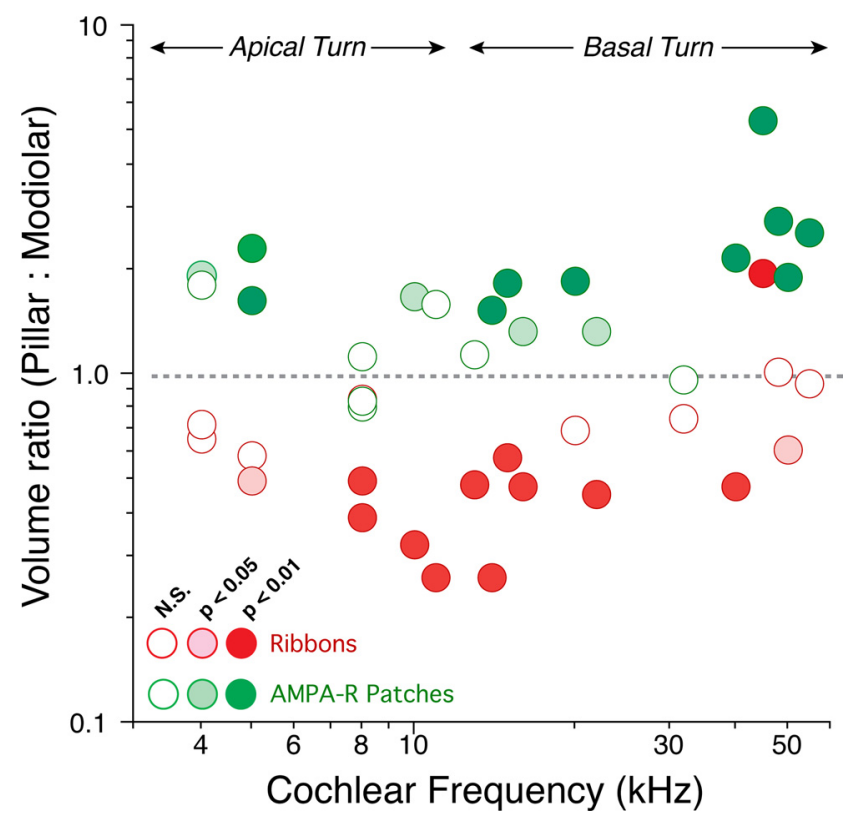

Figure 6. Pillar/modiolar gradient of synaptic morphologies on the sensory cell at different cochlear locations, from apical (low-frequency) to basal (high-frequency) turns. Pooled data from Figure 5 are replotted here to show each cochlear region analyzed. Each confocal $z$-stack produces two data points: one (red) for the ratio between the mean ribbon size on pillar/apical versus modiolar/basal zones of the cell, and one (green) for the ratio between the mean size of AMPAR patches on the two sides of the cell. Fill color indicates the level of statistical significance (unpaired Student's $t$ test comparing the volumes from pillar/apical versus modiolar/basal sides; see key). Approximate extents of the apical and basal turns of the cochlear spiral are indicated. Further technical details can be found in Materials and Methods.

were $\sim 80 \%$ (or $85 \%$ ) bigger in the modiolar/basal zone (Fig. 5 , insets).

Although the spatial segregation shown here is highly significant, the measurements likely underestimate the clarity of the separation: when viewing the IHC row in the $y z$ projection (Fig. $4 B$ ), alignment of IHC basal poles is much less regular than the alignment of their apical surfaces. Indeed, adjacent IHCs of- ten alternate in the distance of their nuclei from the OHCs (supplemental Fig. 4, available at www.jneurosci.org as supplemental material): this staggered misalignment, which may maximize packing density for the relatively bulbous nuclear regions, will degrade the clarity of any spatial separation we can measure in these $y z$ projections.

To investigate possible differences in IHC synaptic organization along the cochlear spiral, we examined mean morphometric data from each $z$-stack (i.e., $4-5$ adjacent IHCs) individually. In the scatter plot of Figure 6, each image stack generates the following two symbols: one (red tones) for the ratio of ribbon volumes (pillar: modiolar); and one (green tones) for the ratio of AMPAR patch volume (pillar/modiolar). The asymmetries in ribbon volume are largest and most significant (see key) in mid-cochlear regions: apical to the $8 \mathrm{kHz}$ place, no image stack showed significant differences at the $p<$ 0.01 level; basal to the $30 \mathrm{kHz}$ place, one sample showed the reverse gradient (pillar $>$ modiolar) and only one of the remaining five samples was significant at the 0.01 level. The asymmetries in AMPAR patch size were present throughout the cochlear spiral; however, the data were less consistent in the apical turn. This difference can be seen by comparing the clarity of synaptic segregation in the $y z$ projection from the basal turn (Fig. $2 \mathrm{~B}$ ) with that from the middle of the cochlear spiral (Fig. $4 B$ ).

\section{Discussion}

\section{Functional subtypes of cochlear nerve fibers}

Each cochlear neuron is excited by a single glutamatergic synapse with a single IHC. This synapse is specialized for precision and speed to subserve a sensory system that uses microsecond timing differences to decode the positions of sounds in space. The ribbon synapse provides presynaptic precision by coordinating vesicle release upon sound-evoked hair cell depolarization (Khimich et al., 2005; Buran et al., 2010). Precision timing is preserved postsynaptically by expression of rapid, AMPA-type GluRs (Matsubara et al., 1996; Grant et al., 2010). Among the four types of AMPA receptors, the GluR3 subunits studied here contribute to these rapid kinetics (for review, see Isaac et al., 2007).

Although all cochlear nerve fibers display precision timing (Johnson, 1980), there are striking differences in their sensitivity to sound, which contribute to the impressive dynamic range of the auditory periphery (Liberman, 1978). In cat, the relation between threshold sensitivity and SR suggested the following three groups of cochlear nerve fibers (Liberman, 1978): high SR ( $>20$ $\mathrm{sp} / \mathrm{s}$ ) fibers with the lowest thresholds (60\% of the sample); low SR $(>0.5 \mathrm{sp} / \mathrm{s})$ fibers with the highest thresholds $(15 \%$ of the sample); and medium SR fibers with intermediate threshold sensitivity (the remaining 25\%). A similar relation between SR and thresholds is seen in guinea pig (Winter et al., 1990), chinchilla (Salvi et al., 1982), gerbil (Schmiedt, 1989), rabbit (Borg et al., 1988), and mouse (Taberner and Liberman, 2005). Although data from other species do not provide the resolution to justify separation of three, rather than two, subgroups, a strict SR- 

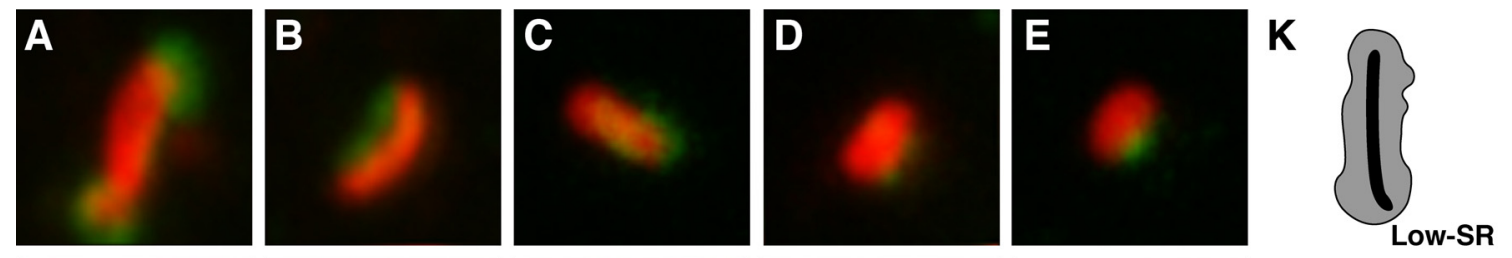

L
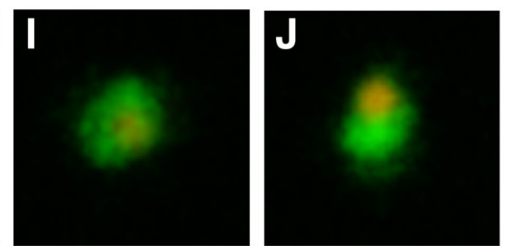

M

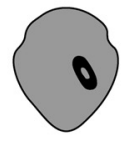

High-SR

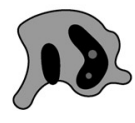

Low-SR

$\mathbf{N}$

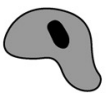

High-SR

Figure 7. High-power confocal views of selected colocalized ribbons and AMPAR patches on modiolar/basal $(\boldsymbol{A}-\boldsymbol{E})$ versus pillar/apical $(\boldsymbol{F}-\boldsymbol{J})$ sides of the hair cell compared with electron microscopic reconstructions of ribbons and postsynaptic densities from identified low-SR $(\boldsymbol{K}, \boldsymbol{L})$ versus high-SR $(\boldsymbol{M}, \boldsymbol{N})$ synapses from an intracellular labeling study of cochlear nerve fibers in cat (Merchan-Perez and Liberman, 1996). Scale bar in $\boldsymbol{F}$ applies to all panels, including those from the ultrastructural study. For display purposes, these images were resampled (bicubic) in Photoshop to minimize pixelization. Quantification was always performed on raw image stacks.

related stratification of threshold sensitivity appears to be a fundamental feature of the mammalian cochlear nerve.

Intracellular labeling studies in both cat (Liberman, 1991) and guinea pig (Tsuji and Liberman, 1997) also show consistent intergroup differences in central projections, which further suggests that different SR groups make different contributions to central auditory processing. Noise-masking studies have shown that high-threshold, low-SR fibers are particularly important to hearing in noisy environments (Costalupes et al., 1984). The fact that low-SR fibers are more vulnerable to degeneration in the aging ear may help explain why hearing in noise is an increasing problem in age-related hearing loss (Schmiedt and Schulte, 1992).

\section{Synaptic segregation on the IHC: modiolar versus pillar or} basal versus apical

The synaptic complexes at the IHC/cochlear nerve junction typically contain a single presynaptic ribbon, surrounded by a compact halo of vesicles and a single discrete plaque of postsynaptic membrane density (Liberman, 1980a; Stamataki et al., 2006), Intracellular labeling in cat and guinea pig showed that fibers from all three SR groups arise from type-I spiral ganglion cells, and that each fiber contacts a single IHC by a single terminal swelling (Liberman, 1982a; Tsuji and Liberman, 1997). Prior ultrastructural work (Liberman, 1980a), based on reconstruction of $\sim 100$ synapses from serial sections, suggested that the peripheral terminals of cochlear nerve fibers are spatially segregated such that low- and medium-SR synapses are found on the modiolar side of the IHC and that high-SR fibers are found on the pillar side (Fig. 1). Subsequent intracellular labeling of 56 physiologically characterized neurons in cat confirmed the overall pattern but showed that high-SR fibers are sometimes found on the modiolar side (Liberman, 1982a). A similar spatial segregation of the different SR groups around the IHC has been demonstrated by intracellular labeling in the guinea pig (Tsuji and Liberman, 1997).

The present results, based on confocal image stacks of 1243 synapses on $>100$ IHCs, suggest a modified view of the spatial gradient; that is, that it may be better described as running from basal to apical pole of the IHC, rather than from its modiolar to its pillar side (Fig. $4 B$ ). In prior ultrastructural studies (Liberman, 1980a), the sectioning angle was parallel to the cuticular plate (Fig. 1). Given the tilt of the IHC body, this section angle (Fig. $4 B$, perpendicular to the solid yellow line) makes the gradient appear pillar versus modiolar. A more recent serial section study of mouse IHCs (Stamataki et al., 2006) describes their cutting angle as "perpendicular to the IHC's long axis": this may explain their failure to observe the same "pillar/modiolar" gradients seen in cat (Liberman, 1980a). If synapses with large receptor patches and small ribbons correspond to high-SR fibers (and vice versa), the image in Figure $4 B$ suggests that high-SR fibers should sometimes be found on the modiolar side of the hair cell, whereas lowand medium-SR fibers should never be found on the pillar side: this is precisely the pattern suggested by prior work (Liberman, 1980a, 1982a). Triple stains with an explicit hair cell marker and a rigorous hair cell-based coordinate system (in addition to labels for ribbons and GluRs) will be required to decide which axes best define the spatial gradients on the IHC.

If the gradient for synaptic heterogeneity is from basal to apical pole, rather than from pillar to modiolar side, the signaling pathways underlying its genesis are likely different from those involved in the development of other types of planar cell polarity (e.g., hair bundle orientation, which runs along a pillar to modiolar axis) (Kelly and Chen, 2009). The olivocochlear efferent bundle could be involved in the development of the synaptic gradient, given that (1) efferent nerves arrive in the IHC area 1-2 $\mathrm{d}$ after birth (Pujol et al., 1978), while the heterogeneity in cochlear nerve SRs first appears between P7 and P20 (Walsh and McGee, 1987); and (2) spiraling efferent bundles run close to the basal pole of the IHC (Liberman, 1980b), such that the distance from developing efferent innervation may be directly related to the size of the mature AMPAR patch.

\section{Cochlear nerve responses and the opposing gradients of ribbons and receptors}

Although spatial segregation of high-SR versus low- and medium-SR fibers around the hair cell was suggested 30 years ago (Liberman, 1980a), none of the prior morphological studies suggested a compelling mechanism for the observed functional differences, for example: (1) it seems unlikely that the membrane potential differs on the two sides of the hair cell; (2) the larger ribbon size and greater tethered vesicle counts for low-SR synapses seem paradoxical; and (3) increased mitochondrial content for high-SR fibers is consistent with increased spike activity (and thus ion flux), however, this difference seems more likely an effect, rather than a cause, of the sensitivity differences.

The present results reveal a complementary spatial gradient in the sizes of postsynaptic AMPAR patches and presynaptic ribbons (Figs. 3-5). The new feature of this spatial organization in 
the hair cell's afferent innervation (i.e., the AMPAR gradient) is likely key to the sensitivity differences among cochlear primary sensory fibers. Although the mean difference between AMPAR patches in the two IHC regions was $64 \%$, the range of sizes in individual image stacks was $>600 \%$ (Fig. 5). The ultrastructural observation that postsynaptic densities are approximately equal in size for low- versus high-SR fibers (Fig. $7 K-N$ ), despite the apparently large size difference in AMPAR patches (Fig. $7 A-J$ ), suggests that other glutamate receptors, including AMPA, kainate, or NMDA, may be expressed in a complementary gradient (i.e., relatively more plentiful at low- than high-SR synapses). With respect to AMPARs, GluR4, but not GluR1, has been localized at cochlear nerve synapses in addition to GluR2/3 (Matsubara et al., 1996; Furness and Lawton, 2003). Metabotropic GluRs, as well as NMDA and kainate receptors, are also expressed in cochlear neurons (Niedzielski and Wenthold, 1995), and NMDA receptors have been localized to puncta at the bases of IHCs (Ruel et al., 2008). Of course, the differences in postsynaptic receptor expression suggested here likely act in concert with presynaptic differences, such as the number of voltage-gated calcium channels clustered at the active zone (Frank et al., 2009), to produce the range of EPSC amplitudes observed among IHC terminals in vitro (Grant et al., 2010), and the heterogeneity in cochlear nerve SRs and thresholds in vivo (Liberman, 1978), as well as to possible differences in the vulnerability to acoustic overstimulation (Kujawa and Liberman, 2009).

Prior ultrastructural analysis found the largest and most complex ribbons on the modiolar face of the IHC (Liberman et al., 1990), and intracellular labeling found these large ribbons opposite low-SR fibers (Merchan-Perez and Liberman, 1996). The present confocal results also show the largest ribbons in the modiolar/basal zone of the IHCs (Figs. $4 B, 5$ ). The en face electron microscopic reconstructions of identified high-SR versus low-SR ribbons (Fig. $7 K-N$ ) are similar in size and shape to the confocal images of putative high-SR versus low-SR ribbons (Fig. $7 A-J$ ), suggesting that $\mathrm{CtBP} 2 /$ ribeye antigenic sites are distributed throughout the ribbon, thereby allowing its full structure to be imaged via immunostaining. Indeed, CtBP2/ribeye is reported to be the major structural component of the ribbon (Schmitz, 2009).

Differences in synaptic ribbon size may contribute to the heterogeneity in EPSC shape seen in cochlear nerve terminals in vitro; whereas, the majority of terminals showed large, monophasic EPSCs, a minority $(\sim 30 \%)$ showed a preponderance of smaller, multiphasic EPSCs (Grant et al., 2010). The multiphasic EPSCs in these putative low- or medium-SR fibers could arise from uncoordinated vesicular release along the extremely elongate ribbons that characterize these synapses. Such elongate ribbons sometimes span multiple AMPAR patches postsynaptically (Fig. $7 A, B$ ) and, thus, might also be long enough to span multiple calcium-channel clusters presynaptically. As such, they may also span multiple $\mathrm{Ca}^{2+}$ microdomains (Frank et al., 2009), resulting in uncoordinated vesicular release from different regions of the ribbon. In contrast, the more compact, spherical ribbons opposite high-SR fibers (Fig. $7 F, G$ ) may favor coordinated vesicle release, and the coupling of these compact ribbons with dense AMPAR clusters, which often encircle the ribbon (Fig. 7G), should further favor rapid synaptic transmission with fast, monophasic EPSCs.

\section{References}

Borg E, Engström B, Linde G, Marklund K (1988) Eighth nerve fiber firing features in normal-hearing rabbits. Hear Res 36:191-201.
Buran BN, Strenzke N, Neef A, Gundelfinger ED, Moser T, Liberman MC (2010) Onset coding is degraded in auditory nerve fibers from mutant mice lacking synaptic ribbons. J Neurosci 30:7587-7597.

Collingridge GL, Olsen RW, Peters J, Spedding M (2009) A nomenclature for ligand-gated ion channels. Neuropharmacology 56:2-5.

Costalupes JA, Young ED, Gibson DJ (1984) Effects of continuous noise backgrounds on rate response of auditory nerve fibers in cat. J Neurophysiol 51:1326-1344.

Frank T, Khimich D, Neef A, Moser T (2009) Mechanisms contributing to synaptic Ca2 + signals and their heterogeneity in hair cells. Proc Natl Acad Sci U S A 106:4483-4488.

Furness DN, Lawton DM (2003) Comparative distribution of glutamate transporters and receptors in relation to afferent innervation density in the mammalian cochlea. J Neurosci 23:11296-11304.

Grant L, Yi E, Glowatzki E (2010) Two modes of release shape the postsynaptic response at the inner hair cell ribbon synapse. J Neurosci 30:4210-4220

Isaac JT, Ashby M, McBain CJ (2007) The role of the GluR2 subunit in AMPA receptor function and synaptic plasticity. Neuron 54:859-871.

Johnson DH (1980) The relationship between spike rate and synchrony in responses of auditory-nerve fibers to single tones. J Acoust Soc Am 68:1115-1122.

Kelly MC, Chen P (2009) Development of form and function in the mammalian cochlea. Curr Opin Neurobiol 19:395-401.

Khimich D, Nouvian R, Pujol R, Tom Dieck S, Egner A, Gundelfinger ED, Moser T (2005) Hair cell synaptic ribbons are essential for synchronous auditory signalling. Nature 434:889-894.

Kujawa SG, Liberman MC (2009) Adding insult to injury: cochlear nerve degeneration after "temporary" noise-induced hearing loss. J Neurosci 29:14077-14085.

Liberman MC (1978) Auditory-nerve response from cats raised in a lownoise chamber. J Acoust Soc Am 63:442-455.

Liberman MC (1980a) Morphological differences among radial afferent fibers in the cat cochlea: an electron-microscopic study of serial sections. Hear Res 3:45-63.

Liberman MC (1980b) Efferent synapses in the inner hair cell area of the cat cochlea: an electron microscopic study of serial sections. Hear Res 3:189-204.

Liberman MC (1982a) Single-neuron labeling in the cat auditory nerve. Science 216:1239-1241.

Liberman MC (1982b) The cochlear frequency map for the cat: Labeling auditory-nerve fibers of known characteristic frequency. J Acoust Soc Am 72:1441-1449.

Liberman MC (1991) Central projections of auditory nerve fibers of differing spontaneous rate, I: Anteroventral cochlear nucleus. J Comp Neurol 313:240-258

Liberman MC, Dodds LW, Pierce S (1990) Afferent and efferent innervation of the cat cochlea: quantitative analysis with light and electron microscopy. J Comp Neurol 301:443-460.

Matsubara A, Laake JH, Davanger S, Usami S, Ottersen OP (1996) Organization of AMPA receptor subunits at a glutamate synapse: a quantitative immunogold analysis of hair cell synapses in the rat organ of Corti. J Neurosci 16:4457-4467.

McLean WJ, Smith KA, Glowatzki E, Pyott SJ (2009) Distribution of the $\mathrm{Na}, \mathrm{K}-\mathrm{ATP}$ ase alpha subunit in the rat spiral ganglion and organ of corti. J Assoc Res Otolaryngol 10:37-49.

Merchan-Perez A, Liberman MC (1996) Ultrastructural differences among afferent synapses on cochlear hair cells: correlations with spontaneous discharge rate. J Comp Neurol 371:208-221.

Meyer AC, Frank T, Khimich D, Hoch G, Riedel D, Chapochnikov NM, Yarin YM, Harke B, Hell SW, Egner A, Moser T (2009) Tuning of synapse number, structure and function in the cochlea. Nat Neurosci 12:444-453.

Müller M, von Hünerbein K, Hoidis S, Smolders JW (2005) A physiological place-frequency map of the cochlea in the CBA/J mouse. Hear Res 202:63-73.

Niedzielski AS, Wenthold RJ (1995) Expression of AMPA, kainate, and NMDA receptor subunits in cochlear and vestibular ganglia. J Neurosci 15:2338-2353.

Pujol R, Carlier E, Devigne C (1978) Different patterns of cochlear innervation during the development of the kitten. J Comp Neurol 177:529-536. 
Ruel J, Wang J, Rebillard G, Eybalin M, Lloyd R, Pujol R, Puel JL (2007) Physiology, pharmacology and plasticity at the inner hair cell synaptic complex. Hear Res 227:19-27.

Ruel J, Chabbert C, Nouvian R, Bendris R, Eybalin M, Leger CL, Bourien J, Mersel M, Puel JL (2008) Salicylate enables cochlear arachidonic-acidsensitive NMDA receptor responses. J Neurosci 28:7313-7323.

Salvi R, Perry J, Hamernik RP, Henderson D (1982) Relationships between cochear pathologies and auditory nerve and behavioral responses following acoustic trauma. In: New perspectives on noise-induced hearing (Hamernik RP, Henderson D, Salvi R, eds), pp 165-188. New York: Raven.

Schmiedt RA (1989) Spontaneous rates, thresholds and tuning of auditorynerve fibers in the gerbil: comparisons to cat data. Hear Res 42:23-35.

Schmiedt RA, Schulte BA (1992) Physiologic and histopathologic changes in quiet- and noise-aged gerbil cochleas. In: Noise induced hearing loss (Dancer AL, Henderson D, Salvi RJ, Hamernik RP, eds), pp 246-258. St. Louis: Mosby.
Schmitz F (2009) The making of synaptic ribbons: how they are built and what they do. Neuroscientist 15:611-624.

Spoendlin H (1969) Innervation patterns in the organ of corti of the cat. Acta Otolaryngol 67:239-254.

Stamataki S, Francis HW, Lehar M, May BJ, Ryugo DK (2006) Synaptic alterations at inner hair cells precede spiral ganglion cell loss in aging C57BL/6J mice. Hear Res 221:104-118.

Taberner AM, Liberman MC (2005) Response properties of single auditory nerve fibers in the mouse. J Neurophysiol 93:557-569.

Tsuji J, Liberman MC (1997) Intracellular labeling of auditory nerve fibers in guinea pig: central and peripheral projections. J Comp Neurol 381:188-202.

Walsh EJ, McGee J (1987) Postnatal development of auditory nerve and cochlear nucleus neuronal responses in kittens. Hear Res 28:97-116.

Winter IM, Robertson D, Yates GK (1990) Diversity of characteristic frequency rate-intensity functions in guinea pig auditory nerve fibres. Hear Res 45:191-202. 